\title{
Minimally Invasive Plate Osteosynthesis with Conventional Compression Plate for Diaphyseal Tibia Fracture
}

\author{
IM Anuar-Ramdhan, M Med Orth, IM Azahari, MD
}

Department of Orthopaedic Surgery, Hospital Sultanah Nur Zahirah, Kuala Terengganu, Malaysia

\begin{abstract}
The diaphyseal tibia fracture is best treated with intramedullary nail but in some cases where the nail is not applicable, plate fixation will be the next option of fixation. The extensile anterior approach is normally used for conventional compression plate fixation in tibia shaft fractures. The extensive surgical dissection may devitalizes the bony fragments and interfere with the fracture union as well as soft tissue healing. Minimally Invasive Plate Osteosynthesis (MIPO) provides good preservation of blood supply and fracture hematoma at the fracture site thus promotes biological bone healing. The use of indirect reduction techniques and small skin incisions to introduce the plate is technically demanding and requires fluoroscopy exposures throughout the surgery, being some of its drawbacks. We recommend MIPO for conventional compression plate fixation in tibial shaft fractures in view of the reduced surgical trauma to the surrounding soft tissue and good functional outcome.
\end{abstract}

\section{Key Words:}

Diaphyseal tibia fracture, conventional compression plate, minimally invasive plate osteosynthesis

\section{INTRODUCTION}

The diaphyseal tibia fracture is best treated with intramedullary nailing. It promotes biological bone healing with preservation of osteogenic fracture hematoma and is mechanically stable with load-sharing principles. However, in certain cases the selection of intramedullary nailing for tibial shaft fracture is inappropriate; for example tibial fracture in children, proximal diaphyseal tibia fracture with ipsilateral avulsion fracture of Posterior Cruciate Ligament (PCL) and distal diaphyseal tibia fracture with ipsilateral ankle injury that requires fixation. These cases may require other methods of fixation, and plating is a good option.

The indications for using Locking Compression Plate (LCP) for tibial fractures are mainly in osteoporotic bone fracture, diametaphyseal fracture and comminuted peri-articular fracture. LCP provides stable construct as internal external fixator, and promotes biological bone healing, coupled with the minimally invasive surgical approach, or Minimally Invasive Plate Osteosynthesis (MIPO) ${ }^{1}$. Generally, fixation of tibial shaft fracture in children and young patient is by using conventional compression plates [or dynamic compression plate (DCP)] that normally use extensile anterior surgical approach. The disadvantages of this extensile approach are loss of fracture hematoma, extensive soft tissue dissection and periosteal stripping that eventually may lead to fracture non-union, wound infection and skin breakdown ${ }^{2}$.

To minimize all the complications in the extensile approach, MIPO method is the best solution. We report two cases of diaphyseal tibia fractures fixed with conventional compression plates by MIPO techniques.

\section{CASE REPORTS}

Case 1

A 16-year old teenage boy had sustained injury to his right knee and leg in motor-vehicle accident (MVA) in September 2013. Clinical assessment revealed swollen right knee with tenderness at mid-right shin with no signs of compartment syndrome. The radiographs showed fracture mid-shaft of right tibia with ipsilateral Posterior Cruciate Ligament (PCL) avulsion fracture. The diagnoses were closed fracture midshaft right tibia and closed avulsion fracture of right PCL. He underwent plating of right tibia (MIPO technique) and screw fixation of the PCL avulsion fracture.

Patient was positioned supine on the operating table. After the usual preparations of the operation site, two small skin incisions were made, about $1 \mathrm{~cm}$ lateral to the anterior tibial crest, proximal and distal to the fracture site. The two incisions were aimed for the most proximal and most distal screw hole of the conventional compression plate. A subcutaneous tunnel was created onto the periosteum of the lateral tibial surface by using bone lever (Fig.2A). Then, the 

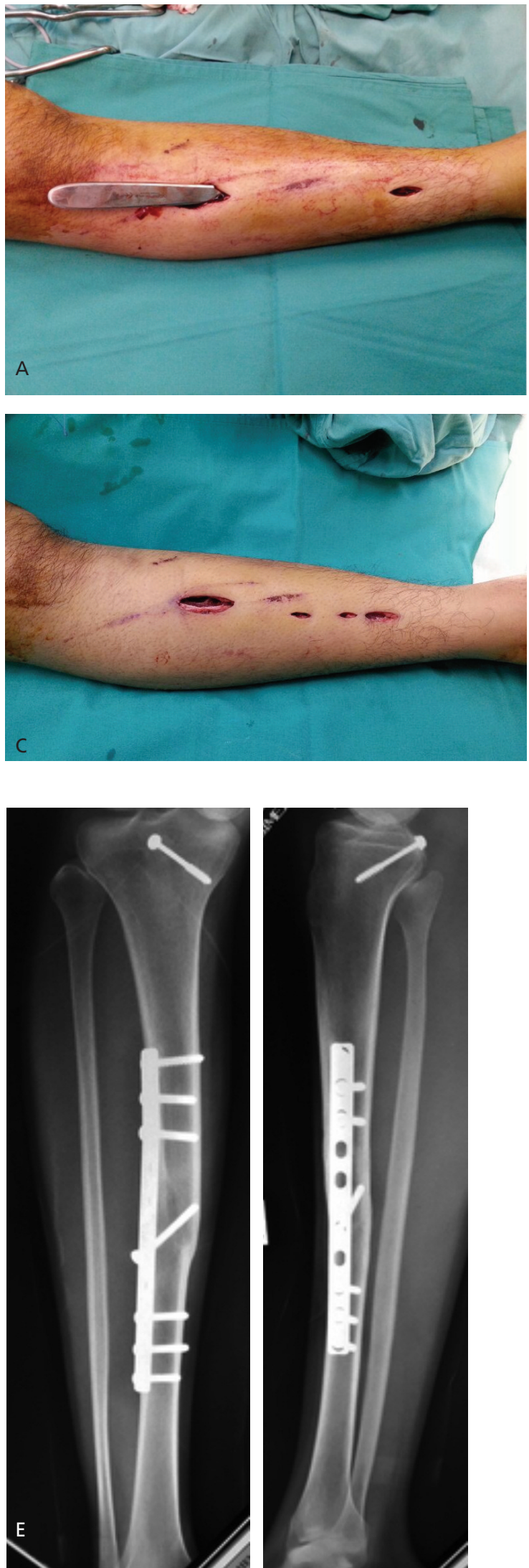

Fig. 2: A. Subcutaneous tunnel created -using bone lever. B. The plate being held temporarily in place with two Kirschner wires. C. Final operative wound with MIPO technique. D. The operative wound healed well. E. The tibial fracture had united well.
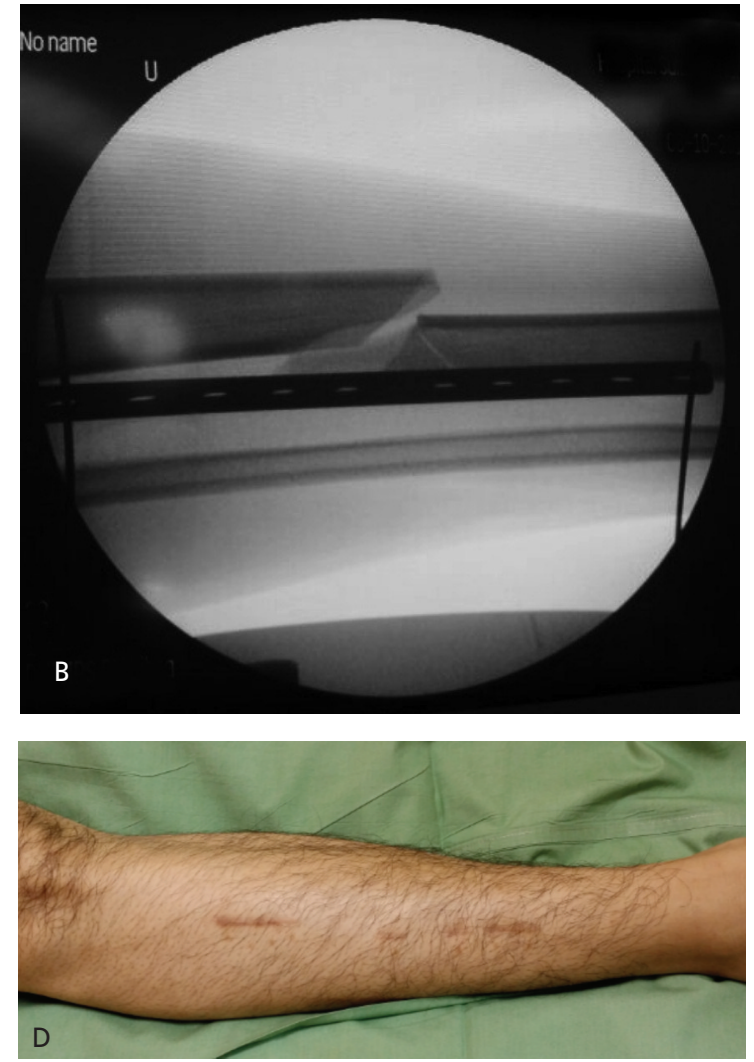

conventional compression plate was introduced and advanced directly onto the periosteum on the lateral surface of the tibia. The periosteum itself was not opened. After the plate was placed in the correct position, it was held temporarily in place with two Kirschner wires for the most proximal and most distal hole of the plate, respectively (Fig.2B). With indirect reduction of the fracture, the plate was fixed to the bone with percutaneously directed screws, and the skin wounds closed after irrigation. (Fig.2C). During the whole procedure fluoroscopic (image intensifier) control was essential.

At follow-up the operative wounds had healed well (Fig.2D) and the tibial fracture had united after five months without any complications (Fig. 2E)

Case 2

A 13-year old boy who was involved in motor-vehicle accident (MVA) in July 2013 had sustained injury to the left leg. There was no other injury. The left leg was deformed and tender with laceration wound over the proximal part of the leg. Neurovascular status was intact. The radiographs showed comminuted fracture proximal third of the left tibia and oblique fracture proximal third of the left fibula (Fig.3A). With the diagnosis of open fracture of the proximal third of the left tibia and fibula, wound debridement and external fixation of the left tibia was performed on Day 2 post-injury, with antibiotic cover as per standard protocol for open fractures. After 6 days post-debridement, definitive surgery was performed: the external fixator was removed 

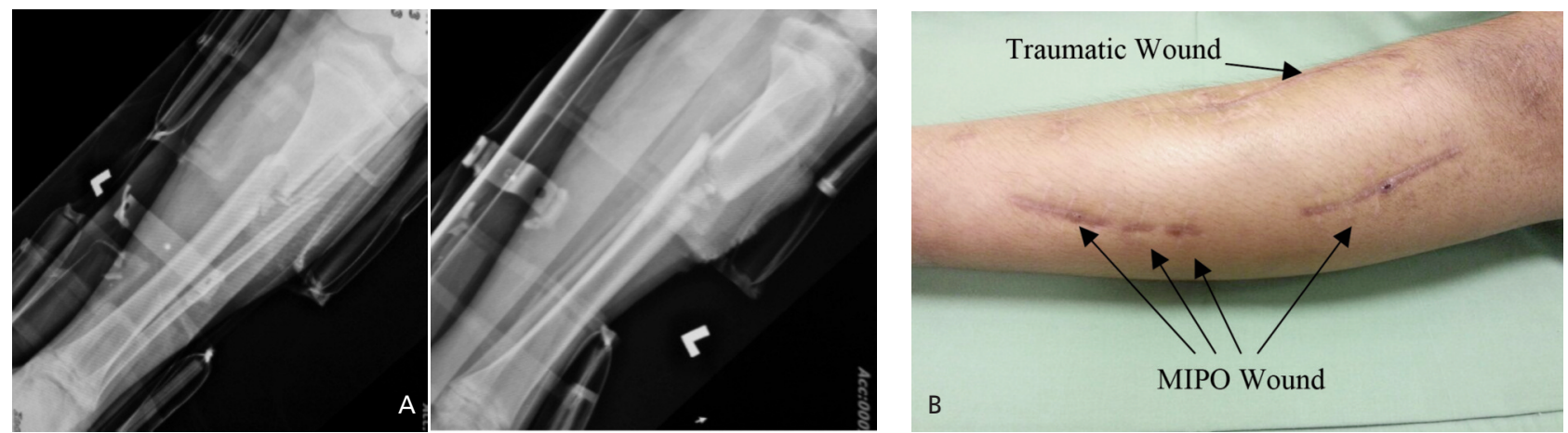
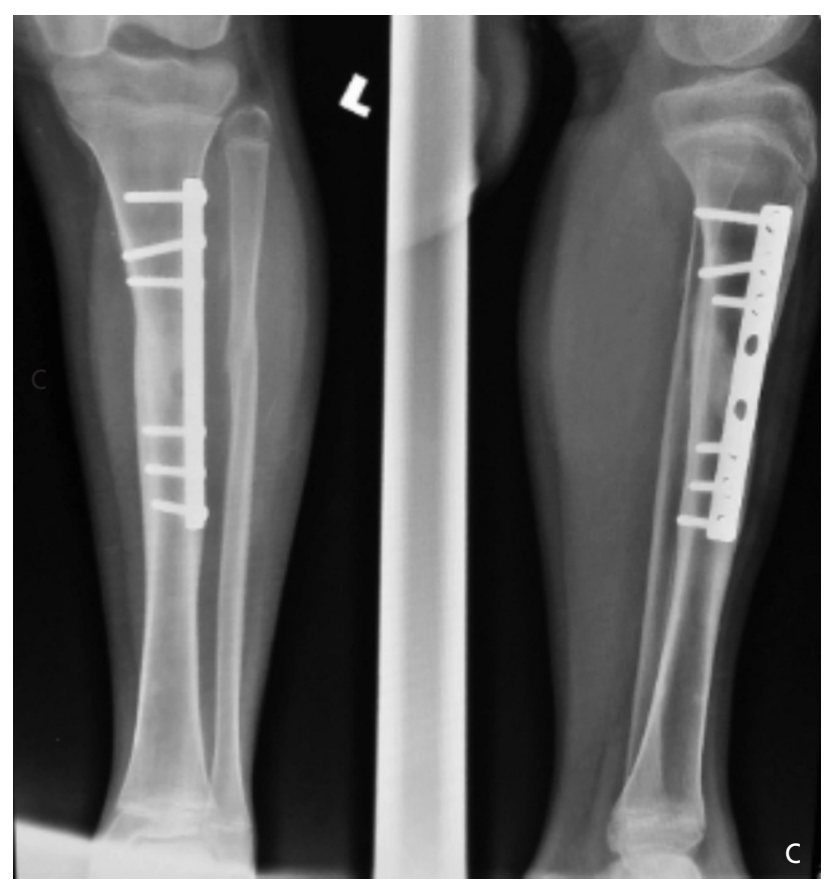

Fig. 3: A. Fracture proximal third of the left tibia and oblique fracture proximal third of the left fibula. B. The operative wound healed well. C. United fractures after 4 month post-MIPO surgery.

and the left tibia was fixed with dynamic compression plate (DCP) using minimallly invasive plate osteosynthesis (MIPO) technique (as described above). He was discharged well on Day 2 post-plating. At review, the operative wounds had healed well (Fig. 3B) and -serial radiographs showed united fracture after four months post-MIPO surgery without any complications. (Fig.3C).

\section{DISCUSSION}

MIPO technique is synonym to the locking compression plate (LCP) fixation, and conventional compression plate is usual with extensile anterior approach of the tibia (for tibial shaft fracture). As MIPO is well known to have many advantages, include preservation of osteogenic potential of the fracture hematoma, biologically friendly, and less surgical trauma to the surrounding soft tissues, it is recommended that all fractures should be preferably be fixed by this MIPO technique ${ }^{1}$.

This less invasive surgery has been described widely for proximal and distal tibial fractures, especially fractures at the diametaphyseal region and peri-articular fractures ${ }^{3-4}$. The outcomes of this techniques showed good wound healing as well as fracture union. There were limited literatures mentioning about MIPO technique for mid-diaphyseal or middle-third tibia shaft fractures, especially lateral placement of the conventional compression plate on the tibia.

Our experience in using MIPO for conventional compression plate in diaphyseal tibia fracture fixation has revealed less soft tissues dissection and periosteal stripping with percutaneous screw placement through smaller surgical wound and less intra-operative bleeding. The amount of sutures for closure of the surgical wound was also minimal which is also cost saving. However, not all tibial shaft fractures are suitable for MIPO technique. Severely displaced comminuted shaft fractures with unstable ipsilateral fibula fracture are among cases that are not suitable to be fixed by MIPO approach. Good selection of cases is important in order to achieve positive outcome of fracture union.

MIPO for diaphyseal tibia fractures can be performed either in closed or open tibial fractures. In closed tibial shaft fractures, there was not much issues in MIPO approach as the skin was nicely covered the fracture. We would be able to plan the skin incision from the beginning unlike in open tibial shaft fractures. The traumatic laceration wound and post-debridement wound in open fractures gives the surgeon a challenge to plan the skin incision for MIPO. In case 2 described above, the traumatic wound was at the anteromedial aspect of the leg. Therefore we needed to make a long curvilinear skin incision in order to achieve $5 \mathrm{~cm}$ skin bridge (to reduce the risk of skin necrosis) (Fig 3B). 
The placement of the plate requires additional skills, as the exposure was minimal. The use of Kirschner wires as temporary fixation was essential to hold the plate at the correct position before inserting the screws. Unlike the LCP that has threaded sleeve to hold the plate close to the bone, the conventional plate needs manual pushing to ensure good contact between the plate and the bone before placing the screws. The plate-bone contact was important for the conventional compression plate as it provides stability to the plate fixation.

This MIPO technique has some drawbacks as well. As it requires indirect reduction of the fracture, there are risks of mal-reduction and mal-alignment which subsequently may cause mal-union ${ }^{4}$. Severe mal-union in tibial shaft fractures may deviate the mechanical axis of the lower limb causing imbalance in force distribution to the adjacent joints, with adverse joint effects. Furthermore, with the small skin incisions the insertion of the plate is technically demanding and also requires strict fluoroscopic control throughout the procedure leading to greater radiation exposure compared to open extensile surgical approach ${ }^{5}$.

In conclusion, tibial shaft fractures are best treated with intramedullary nailing but in some cases where this is not applicable, the use of conventional plate with MIPO technique may give good results for bone union as well as soft tissue healing. The smaller surgical wounds also lessen the pain around the operation site and thus accelerate postoperative rehabilitation and faster recovery. We recommend use of MIPO technique for conventional compression plate fixation in tibial shaft fractures.

\section{REFERENCES}

1. Wagner M, Frenk A, Frigg R. Locked plating: Biomechanics and biology and locked plating: Clinical indications. Techniques in Orthopaedics. 2007; 22(4): 209-18.

2. Vallier HA, Le TT, Bedi A. Radiographic and clinical comparisons of distal tibia shaft fractures (4 to $11 \mathrm{~cm}$ proximal to the plafond): plating versus intramedullary nailing. J Orthop Trauma. 2008; 22(5): 307-11.

3. Shrestha D, Acharya B, Shrestha P. Minimally invasive plate osteosynthesis with locking compression plate for distal diametaphyseal tibia fracture. Kathmandu Univ Med J. 2011; 9(2): 62-8.

4. Gupta RK, Rohilla RK, Sangwan K, Singh V, Walia S. Locking plate fixation in distal metaphyseal tibial fractures: series of 79 patients. Int orthop. 2010; 34(8): 1285-90.

5. Francois J, Vandeputte G, Verheyden F, Nelen G. Percutaneous plate fixation of fractures of the distal tibia. Acta orthop belg. 2004; 70(2):148-54 